Annales Geophysicae (2001) 19: 945-952 (C) European Geophysical Society 2001

\title{
Energetics of small scale turbulence in the lower stratosphere from high resolution radar measurements
}

\author{
J. Dole, R. Wilson, F. Dalaudier, and C. Sidi \\ Service d'Aéronomie, Université Pierre et Marie Curie, Paris, France \\ Received: 01 August 2000 - Revised: 02 April 2001 - Accepted: 26 April 2001
}

\begin{abstract}
Very high resolution radar measurements were performed in the troposphere and lower stratosphere by means of the PROUST radar. The PROUST radar operates in the UHF band ( $961 \mathrm{MHz})$ and is located in St. Santin, France $\left(44^{\circ} 39^{\prime} \mathrm{N}, 2^{\circ} 12^{\prime} \mathrm{E}\right)$. A field campaign involving high resolution balloon measurements and the PROUST radar was conducted during April 1998. Under the classical hypothesis that refractive index inhomogeneities at half radar wavelength lie within the inertial subrange, assumed to be isotropic, kinetic energy and temperature variance dissipation rates were estimated independently in the lower stratosphere. The dissipation rate of temperature variance is proportional to the dissipation rate of available potential energy. We therefore estimate the ratio of dissipation rates of potential to kinetic energy. This ratio is a key parameter of atmospheric turbulence which, in locally homogeneous and stationary conditions, is simply related to the flux Richardson number, $R_{f}$.
\end{abstract}

Key words. Meteorology and atmospheric dynamics (turbulence) - Radio science (remote sensing)

\section{Introduction}

It is well known that, in clear air conditions, the backscattered radar echo power is proportional to the 3D spectrum of the refractive index fluctuation field for the radial wavenumber, corresponding to twice the incident wavenumber (Tatarski, 1961; Ottersten, 1969). If specular reflection is negligible, as it may be for wavelengths less than one meter, the refractive index irregularities at the considered scale (15 $\mathrm{cm}$ for the PROUST radar) are assumed to be due to isotropic inertial turbulence only. Under the assumption of local homogeneity and stationarity of the refractive index fluctuations, and by using additional measurements of temperature and humidity gradients (usually balloon borne in-

Correspondence to: R.Wilson

(richard.wilson@aero.jussieu.fr) struments), it is possible to estimate some key parameters of inertial isotropic turbulence (dissipation rate of kinetic energy or refractive index structure constant). However, a major difficulty in radar experiments results from the fact that, for most measurements, the assumption of homogeneity and stationarity of turbulence within the illuminated volume are hardly satisfied. Atmospheric turbulence is known to occur in thin layers (say $100 \mathrm{~m}$ depth or less) and to be intermittent in time.

The main characteristic of the PROUST radar is its very high resolution. The large Cassegrain antenna used $\left(2000 \mathrm{~m}^{2}\right)$ gives an angular resolution of about $0.3^{\circ}$ in the E-W direction, and of $1.1^{\circ}$ in the N-S direction (Half PowerFull Width) (Bertin et al., 1986). The horizontal dimensions of the radar volume are thus about $100 \mathrm{~m}$ and $200 \mathrm{~m}$ in the $\mathrm{E}-\mathrm{W}$ and $\mathrm{N}-\mathrm{S}$ directions, respectively. A pulse compression technique (Petitdidier et al., 1985) provides a $30 \mathrm{~m}$ range resolution. The integration time is reduced to $51 \mathrm{~s}$. Because the turbulent volume is believed to be larger than the radar volume resolution, the assumption of local homogeneity and stationarity of turbulence properties appear reasonable with such a temporal and spatial resolution.

The PROUST radar is thus well suited for turbulence measurements in the upper troposphere and lower stratosphere (Delage et al., 1997). We therefore estimate, by two independent methods, the dissipation rates of available potential energy and kinetic energy. The ratio of these two quantities is known as the mixing efficiency (e.g. Gargett and Moum, 1995). The mixing efficiency is the ratio of irreversible potential energy increase to the dissipation rate of kinetic energy into heat.

Inertial turbulence in the atmosphere is usually characterized either from the structure constant of refractive index fluctuations, $C_{n}^{2}$, or from the dissipation rate of kinetic energy, $\epsilon_{k}$. The structure constant $C_{n}^{2}$, from which is estimated the dissipation rate of potential energy, is evaluated from the backscattered echo power (Gage et al., 1980). The dissipation rate of turbulent kinetic energy, $\epsilon_{k}$, is estimated independently from the broadening of the radar backscatter Doppler 
spectrum (Hocking, 1983, 1985).

In Sect. 2 we review some basic relationships of the energy budget of turbulence in a stratified medium. In Sect. 3 we describe the methods used for extracting dissipation rates from radar measurements. In Sect. 4, the data processing is presented. The results are shown and discussed in Sect. 5. Conclusions are summarized in Sect. 6.

\section{Energy budget of inertial turbulence in a stratified medium}

Under the Boussinesq approximation and by neglecting the transport terms, the turbulent kinetic energy equation becomes (e.g. Monin and Yaglom, 1975)

$$
\frac{\partial E_{k}}{\partial t}=-\overline{u^{\prime} w^{\prime}} \frac{\partial \bar{u}}{\partial z}+\overline{w^{\prime} \theta^{\prime}} \frac{g}{\bar{\theta}}-\epsilon_{k}=P-B-\epsilon_{k}
$$

where $E_{k}$ is the turbulent kinetic energy per unit mass $\left(\mathrm{m}^{2} \mathrm{~s}^{-2}\right), u$ and $w$ are the horizontal and vertical velocities respectively, $\theta$ is the potential temperature. Primes and overbars denote fluctuating and averaged quantities respectively. $P$ and $B$ terms are, respectively, the shear production term and buoyancy conversion term.

With the same assumptions, the equation describing the time evolution of half temperature variance is

$$
\frac{1}{2} \frac{\partial \overline{\theta^{\prime 2}}}{\partial t}=-\overline{u^{\prime} \theta^{\prime}} \frac{\partial \bar{\theta}}{\partial z}-\epsilon_{\theta}
$$

where $\epsilon_{\theta}\left(\mathrm{K}^{2} \mathrm{~s}^{-1}\right)$ is the dissipation rate of half the temperature variance. In a stratified medium, temperature fluctuations are associated with vertical displacements and thus with the available potential energy, $E_{a}$

$E_{a}=\frac{1}{2} \omega_{B}^{2} \overline{\xi^{2}}=\frac{1}{2} \frac{g^{2}}{\omega_{B}^{2}} \overline{\left(\frac{\theta^{\prime}}{\bar{\theta}}\right)^{2}}$

where $\omega_{B}$ is the Brunt-Väisälä frequency $(\mathrm{rad} / \mathrm{s})$ and $\xi$ the vertical displacement. The dissipation rate $\epsilon_{\theta}$ is therefore related to the dissipation rate of available potential energy through:

$\epsilon_{p}=\frac{g^{2}}{\omega_{B}^{2} \bar{\theta}^{2}} \epsilon_{\theta}=\frac{g / \bar{\theta}}{\partial \bar{\theta} / \partial z} \epsilon_{\theta}$

If we also assume steady state turbulent motions, the energy budget reads

$\epsilon_{k}=P-B$ and $\epsilon_{p}=B$

the ratio of these dissipation rates, known as the mixing efficiency, being

$\frac{\epsilon_{p}}{\epsilon_{k}}=\frac{B}{P-B}=\frac{R_{f}}{1-R_{f}}$

where $R_{f}=B / P$ is the flux Richardson number.

The heat diffusion coefficient, defined as the heat flux per unit gradient, can be expressed directly from the dissipation rate of potential energy (Eqs. 2 and 4)

$K_{\theta}=\frac{\epsilon_{p}}{\omega_{B}^{2}}$

\section{Estimation of dissipation rates from radar measure- ments}

\subsection{Dissipation rate of kinetic energy}

The method described by Hocking (1983) relates the dissipation rate of kinetic energy $\epsilon_{k}$ to the width of the Doppler spectrum (second moment of the radar Doppler spectrum). Assuming isotropic inertial fluctuations, the mean square of the turbulent half-velocity variance $\overline{v^{\prime 2}}$ is

$\frac{1}{2} \overline{v^{\prime 2}}=\int_{k_{B}}^{k_{\lambda}} F(k) d k+\int_{-k_{\lambda}}^{-k_{B}} F(k) d k$

where $k_{B}$ and $k_{\lambda}$ are the wavenumber associated with the buoyancy length and half radar wavelength, respectively. $F(k)$ is the 1D "measurable" longitudinal spectrum (i.e. the spectrum of a data sequence of the velocity component along the probing direction):

$F(k)=\alpha \epsilon_{k}^{2 / 3} k^{-5 / 3}$

where $\alpha \approx 0.25$. Integration of (8), by assuming $k_{B} \ll k_{\lambda}$, brings:

$\epsilon_{k} \approx 0.54\left(\overline{v^{\prime 2}}\right)^{3 / 2} k_{B}$

Weinstock (1978) has suggested expressing $k_{B}$ as

$k_{B} \approx 0.62 \frac{\omega_{B}^{3 / 2}}{\epsilon_{k}^{1 / 2}}$

The dissipation rate of kinetic energy is thus evaluated by

$\epsilon_{k} \approx 0.5 \overline{v^{\prime 2}} \omega_{B}$

Contributions to spectral broadening, other than turbulence, can also be important if not dominant (beam or shear broadening, gravity waves or 2D turbulence contamination). However the PROUST radar is well suited for this method thanks to its exceptional angular, vertical and temporal resolution as well as its vertical line of sight. A transverse $\mathrm{N}-\mathrm{S}$ wind of say $10 \mathrm{~m} / \mathrm{s}$ will produce a broadening of about $0.2 \mathrm{~Hz}$. The beam broadening due to the transverse wind was estimated by using an averaged wind profile obtained from the three instrumented balloons. The resulting broadening, about $0.15 \mathrm{~Hz}$, is less than the spectral resolution $(0.2 \mathrm{~Hz})$, and was therefore neglected. The mean squared turbulent velocity $\overline{v^{\prime 2}}$ is thus assumed to be simply related to the width of the Doppler spectrum

$\overline{v^{\prime 2}}=\left(\frac{\lambda_{r}}{2}\right)^{2} \sigma_{f}^{2}$

where $\sigma_{f}$ is the rms width $(\mathrm{Hz})$ of the atmospheric echo spectrum and $\lambda_{r}$ the radar wavelength. 


\subsection{Dissipation rate of available potential energy}

The radar equation relates backscattered power at the radar antenna, $P_{r}$, to the reflectivity per unit volume $\eta$ of the illuminated volume (Doviak and Zrnić, 1984):

$P_{r}=P_{t} \frac{L \lambda_{r}^{2} G_{B}}{16 \pi^{2}} \frac{\Delta r}{r^{2}} \eta$

where $G_{B}$ is the two-way antenna gain $\left(G_{B}=\right.$ $\int G(\theta, \phi)^{2} \mathrm{~d} \Omega / 4 \pi$, the other parameters being described in Table 1. This method requires a well calibrated radar. The PROUST radar has been calibrated from the known noise power which is essentially of instrumental origin, the cosmic noise being very weak at the radar frequency $(961 \mathrm{MHz})$.

Within the inertial subrange, assumed locally homogeneous and isotropic, the radar reflectivity is related to the structure constant of refractive index $C_{n}^{2}$ (Tatarski, 1961)

$\eta=0.38 \lambda_{r}^{-1 / 3} C_{n}^{2}$

where

$C_{n}^{2}=a^{2} \frac{\epsilon_{n}}{\epsilon_{k}^{1 / 3}}$

$\epsilon_{n}$ being the dissipation rate of half the refractive index variance and $a^{2}$ is a constant ( $a^{2} \approx 3.48$ ). If humidity is negligible (i.e. in the stratosphere), $C_{n}^{2}$ is simply proportional to $C_{T}^{2}$

$C_{n}^{2}=\left(0.776 \cdot 10^{-6} \frac{P}{T^{2}}\right)^{2} C_{T}^{2}$

where $P$ is the pressure $(\mathrm{Pa})$, and $T$ the temperature $(\mathrm{K})$. Expressing $C_{T}^{2}$ as a function of the dissipation rate of the temperature variance $\epsilon_{\theta}$ yields :

$C_{T}^{2}=a^{2} \frac{\epsilon_{\theta}}{\epsilon_{k}^{1 / 3}}$

The $C_{n}^{2}$ averaged on the radar volume is evaluated from the backscattered power (Eqs. 14 and 15). By using additional pressure and temperature data, $C_{T}^{2}$ is deduced (Eq. 17) (humidity is assumed negligeable in the lower stratosphere). From Eqs. (4) and (18) we therefore estimate the dissipation rate of available potential energy:

$\epsilon_{p}=\frac{g^{2} / \bar{\theta}^{2}}{\omega_{B}^{2} a^{2}} C_{T}^{2} \epsilon_{k}^{1 / 3}$

where the radar estimate of $\epsilon_{k}$ (Eq. 12) is used. The $\epsilon_{p}$ estimate is, however, weakly dependent on $\epsilon_{k}$, the latter appearing at the one-third power in Eq. (19).

\section{Data description and processing}

The PROUST ST radar, located at St. Santin, France (4439' N, 2 ${ }^{\circ} 12^{\prime}$ E), is a UHF (961 MHz) pulsed Doppler radar. Radar measurements consist of wind vertical velocity, Doppler width and reflectivity profiles in the $3-16 \mathrm{~km}$ range. The main characteristics of the PROUST radar are summarized in Table 1.
Table 1. PROUST radar parameters

\begin{tabular}{lll}
\hline Symbol & Parameter & Value \\
\hline$f$ & Radar frequency & $961 \mathrm{MHz}$ \\
$\lambda_{r}$ & Wavelength & $0.31 \mathrm{~m}$ \\
$P_{t}$ & Peak power & $26 \mathrm{~kW}$ \\
$L$ & Loss term & 0.37 \\
$A_{e}$ & Antenna area & $100 \times 20 \mathrm{~m}^{2}$ \\
$\tau$ & Pulse length & $4 \mu \mathrm{s}$ \\
$\tau_{p}$ & Coded pulse length & $0.2 \mu \mathrm{s}$ \\
$\Delta r$ & Range resolution & $30 \mathrm{~m}$ \\
$F_{r}$ & Pulse repetition frequency & $6.4 \mathrm{kHz}$ \\
$r$ & Range & $3-16 \mathrm{~km}$ \\
$T_{I P}$ & Inter-pulse period & $156.2 \mu \mathrm{s}$ \\
$N_{\text {coh }}$ & Coherent integrations & 128 \\
$N_{\text {code }}$ & Number of code & 20 \\
$N_{\text {inco }}$ & Incoherent integrations & 10 \\
$\Delta t$ & Temporal resolution & $51 \mathrm{~s}$ \\
$B_{r}$ & Bandwidth of the receiver & $5 \mathrm{MHz}$ \\
$T_{N}$ & Equivalent noise temperature & $250 \mathrm{~K}$ \\
& at the receiver output & \\
\hline
\end{tabular}

\subsection{Radar data processing}

After demodulation, the complex signal is Fourier transformed yielding the power spectral density in the $\pm 25.6 \mathrm{~Hz}$ interval with a frequency resolution of $0.2 \mathrm{~Hz}$. Following 10 incoherent integrations of the spectra, the noise level is evaluated and subtracted. The first three moments of the Doppler spectrum (signal power, vertical wind and spectral width) are estimated by fitting the spectrum with a Gaussian function. The data processing of the PROUST radar was described in Delage (1996).

The PROUST radar is calibrated from the known cosmic and instrumental noise. At the radar wavelength, the cosmic noise temperature ranges between 30 and $50 \mathrm{~K}$. After verifying that the noise level on the spectrum (in numerical unit) is consistent with the expected noise at the output of the receiver, the received signal power is estimated from the signal to noise ratio (SNR).

Assuming the observed volume to be filled with scatterers, the averaged refractive index structure constant $C_{n}^{2}$ over the illuminated volume is expressed as a function of the SNR:

$C_{n}^{2}=\frac{16 \pi^{2}}{0.38} \frac{k T_{N} B_{r}}{P_{t} \lambda_{r}^{5 / 3} G_{B} L N_{\text {coh }} N_{\text {code }} H\left(2 k_{\lambda} l_{o}\right)} \frac{r^{2}}{\Delta r} \frac{S}{N}$

where $k$ is the Boltzmann constant, the other terms being defined in Table 1 . The factor $H\left(2 k_{\lambda} l_{0}\right)$ takes into account the departure of the temperature spectrum from the $-5 / 3$ power law for scales close to the inner scale $l_{0}$ of temperature (Hill, 1978a) $\left(l_{0}=7.4\left(v^{3} / \epsilon_{k}\right)^{1 / 4}\right.$ where $v$ is the kinematic viscosity). Since the inner scale for velocity fluctuations is different from $l_{0}$, there exists a viscous-convective subrange. The temperature spectrum in this subrange was described by Hill (1978b), an analytical approximation being given by Frehlich (1992). The induced correction to the classical Kolmogorov 
spectrum at the considered scale $\left(\lambda_{r} / 2\right)$ is evaluated by using the radar estimated $\epsilon_{k}$ (Eq. 12) and is found to be about 1.4. As the range of interest $(10-15 \mathrm{~km})$ is still within the near field of the Cassegrain antenna, the two way gain, $G_{B}$, has to be numerically calculated and tabulated. The antenna gain is found to be a function of altitude, approximately proportional to the range $r$ within the considered altitude domain.

The minimum detectable $C_{n}^{2}$ is of the order of $3 \cdot 10^{-18} \mathrm{~m}^{-2 / 3}$ corresponding to a minimum SNR of about $-13 \mathrm{~dB}$. The spectral resolution being $0.2 \mathrm{~Hz}$, the detectable standard deviation of wind fluctuations is about $0.03 \mathrm{~m} / \mathrm{s}$, corresponding to a minimum $\epsilon_{k}$ of about $10^{-5} \mathrm{~m}^{2} \mathrm{~s}^{-3}$.

\subsection{In situ temperature and wind measurements}

During the continuous radar measurements, seven instrumented balloons were launched from a close site, about $40 \mathrm{~km}$ East of the radar site (dominant wind is directed westward). Every gondola carried a Väisälä RS80G radiosonde including a GPS (Global Positioning System) transponder, thus allowing mesoscale temperature and horizontal wind estimation. Three of the gondolas, referred as SFT (Structure Fine de Température) carried high resolution temperature and pressure sensors, allowing the determination of the gondola altitude by integration of the hydrostatic equation. The temperature sensors are pointed upwind by a vane in order to avoid the wake from the gondola. The high resolution temperature profile has a vertical resolution better than $20 \mathrm{~cm}$ and a noise level corresponding to $3-4 \mathrm{mK}$. Three balloons were launched during each of the first two nights, with a time interval of about 3 hours, the SFT gondola being launched between the two standard Väisälä soundings.

\section{Results and discussion}

Using high resolution temperature and pressure measurements, a profile of refractivity was calculated and its spectrum for the altitude range $11.5-15 \mathrm{~km}$ was estimated. The two spectra displayed on Fig. 1 correspond to two temperature channels of the SFT gondola. The spectral estimation is an average of local spectra estimated on much shorter ( $40 \mathrm{~m}$ ) sections. Because of the intermittent structure of the temperature profile, the average spectrum is dominated by the most turbulent sections in the inertial subrange. Consequently, it is representative of the average level of refractivity fluctuations (and thus $C_{n}^{2}$ ) in the considered altitude range. A description of previous in-situ measurements, using similar techniques, was given in Dalaudier et al. (1994). A sample of the high resolution balloon data is displayed in Fig. 5 along with the corresponding profile for the local $C_{n}^{2}$ estimation.

The vertical spectra shown on Fig. 1 display an inertial subrange, with a slope close to $-5 / 3$ spanning about half a decade, between strong slope (close to -3 ) at large scales, and instrumental noise at small scales. Spurious oscillations are also visible (as narrow peaks), mainly in the high frequency range. The theoretical spectra corresponding to

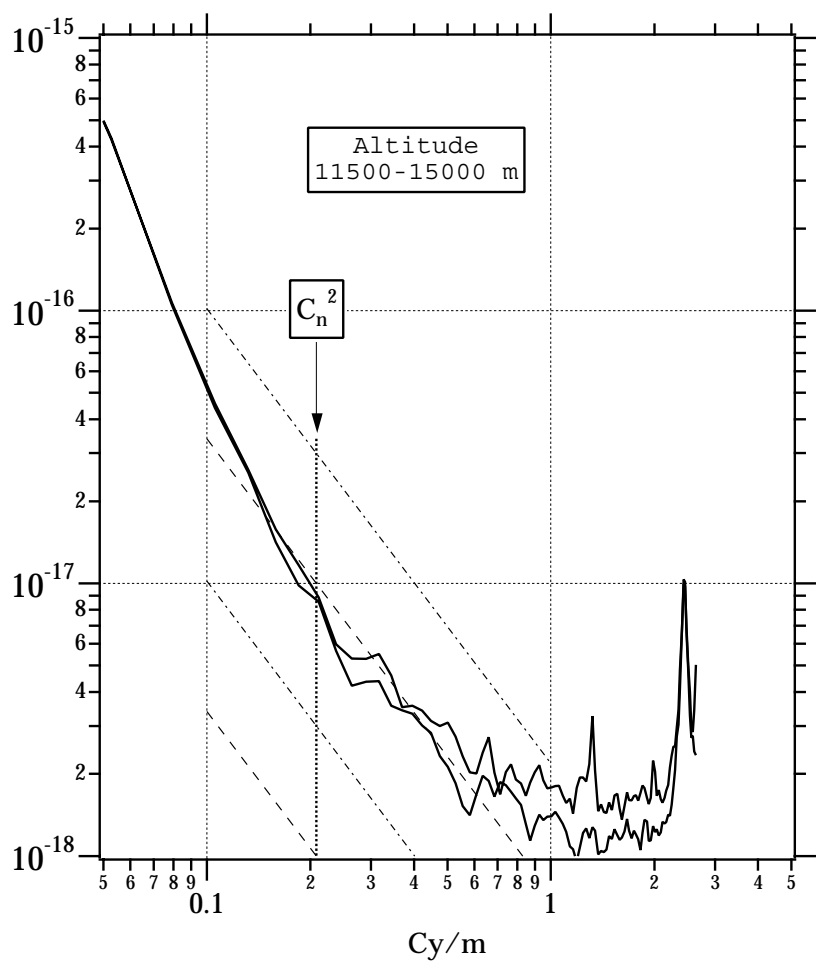

Fig. 1. Refractivity spectrum in the $11.5-15 \mathrm{~km}$ altitude range obtained from high resolution in-situ temperature and pressure measurements on 27 April. The wavenumber for which the spectrum is numerically equal to the mean $C_{n}^{2}$ value is shown as a vertical dotted line.

isotropic inertial range with $-5 / 3$ slopes were also drawn (slant lines) for $C_{n}^{2}$ values of $10^{-18}$ and $10^{-17}$ (dashed) and $3 \cdot 10^{-18}$ and $3 \cdot 10^{-17}$ (dot-dashed) in units of $\mathrm{m}^{-2 / 3}$ according to the formula (Tatarski, 1961):

$S(\beta)=\frac{3}{5} \frac{C_{n}^{2} \beta^{-5 / 3}}{(2 \pi)^{2 / 3} \Gamma(-5 / 3)}=0.073 C_{n}^{2} \beta^{-5 / 3}$

for the one dimensional (observable or folded) turbulent spectrum. Following standard usage, the wavevector $k$ (in $\mathrm{rad} / \mathrm{m})$ is replaced by the wavenumber $\beta=k /(2 \pi)(\mathrm{Cy} / \mathrm{m})$, and the spectrum is normalized accordingly. The wavenumber $\beta^{*}=0.073^{3 / 5}=0.2081 \mathrm{Cy} / \mathrm{m}$, for which the spectrum is numerically equal to the $C_{n}^{2}$ value, is shown as a vertical dotted line. The mean estimate of $C_{n}^{2}$ for the considered altitude range is observed to be close to $10^{-17} \mathrm{~m}^{-2 / 3}$.

In Fig. 2,We show the height-time distribution of $C_{n}^{2}$ obtained from radar measurements between 11.5 and $15 \mathrm{~km}$ during 3 hours on 27 April 1998. The averaged $C_{n}^{2}$ as a function of altitude (averaged on the turbulent patches at a given altitude, not a time average) is shown in the right-hand panel. The $C_{n}^{2}$ estimates range between $3.7 \cdot 10^{-18}$ and $1.8 \cdot 10^{-16}$, the mean value being $2 \cdot 10^{-17} \mathrm{~m}^{-2 / 3}$. Thus a reasonable agreement between in situ and radar estimates of $C_{n}^{2}$ provides an indirect validation of the radar calibration and data processing.

The height-time distribution of $C_{n}^{2}$ in the $11-14.5 \mathrm{~km}$ 

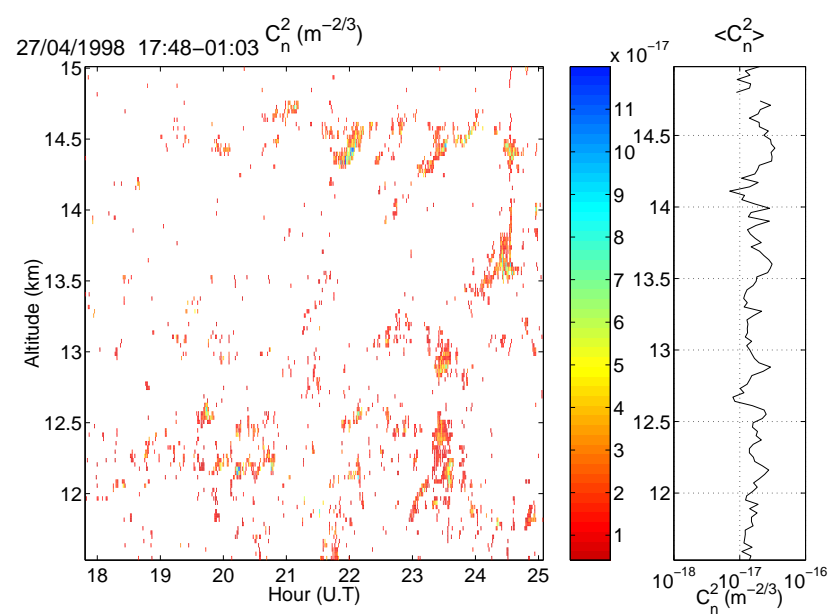

Fig. 2. Left panel: Height-Time distribution of $C_{n}^{2}$ on 27 April 1998. Right plot: Averaged $C_{n}^{2}$ within the turbulent patches.

range for 29 April, is shown on Fig. 3. For this data set, the $C_{n}^{2}$ estimates range between $3.4 \cdot 10^{-18}$ and $5 \cdot 10^{-16}$, the mean value being $4 \cdot 10^{-17} \mathrm{~m}^{-2 / 3}$. Compared to previous data sets, either from PROUST or from balloon measurements, the turbulent activity appears rather weak in the lower stratosphere during these two days.

The turbulent echoes, shown on Figs. 2 and 3 appear to be very sporadic, contrary to VHF echos in the lower stratosphere (e.g. Sato and Woodman, 1982). The cause of this apparent discrepancy with previously published data maybe twofold; first, a threshold effect very likely limits the detection of small reflectivity patches; second, the VHF radar usually have much larger time-space resolution than the PROUST radar and the echoes are then smoothed.

Two Väisälä soundings were performed at the beginning and at the end of the displayed period. The averaged $\omega_{B}$ profile from these two soundings is assumed to be representative of the stratification conditions for a given altitude range during the considered period.

We investigate the dependency of the dissipation rate distributions versus stratification. Figure 4 shows the scatter plot of $\epsilon_{k}$ versus $\epsilon_{p}$ in case of weak stratification (circles) and large stratification (stars) for the 27 April data set. In case of high stability $\left(\omega_{B}^{2}>4 \cdot 10^{-4} \mathrm{~s}^{-2}\right)$ we observe a mean ratio $\left\langle\epsilon_{p} / \epsilon_{k}\right\rangle$ of about 0.1 , as well as a linear fit slope close to one (in a log-log scale), indicative of proportionality. In case of weak stratification $\left(\omega_{B}^{2}<10^{-4} \mathrm{~s}^{-2}\right)$, the potential energy dissipation rate is about one order or magnitude larger than in the previous case, whereas the distribution of kinetic energy dissipation rates appears rather similar.

We think that such an enhancement is a spurious effect owing to the fact that the weak $\omega_{B}$ (average of the two Väisälä soundings) is not representative of stratification within turbulent layers. Temperature profiles resulting from balloons measurements (SFT and Väisälä), as well as a $C_{n}^{2}$ profile obtained from SFT measurements in the 13.6-15.2 altitude range are shown in Fig. 5. In this figure, the diamonds show

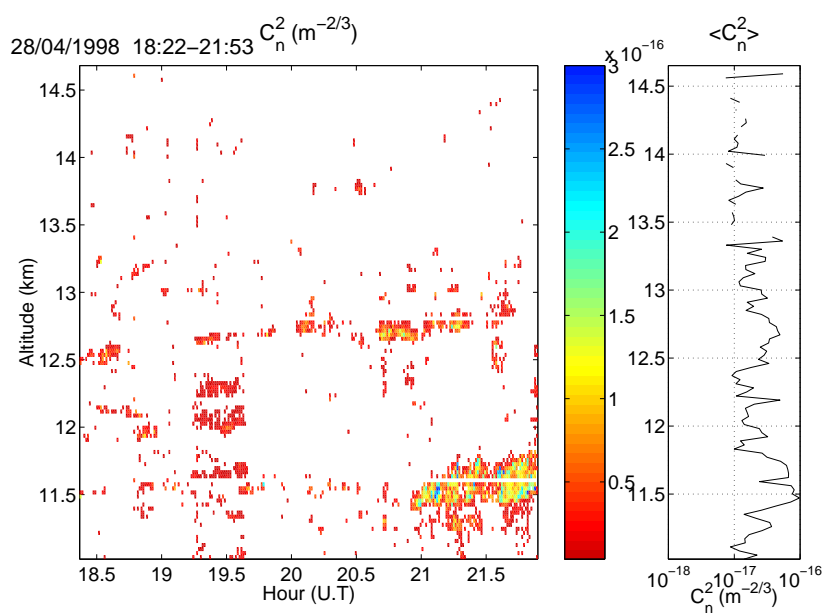

Fig. 3. Same as Fig. 2 but for 28 April 1998.

the standard Väisälä profile resulting from a $10 \mathrm{~s}$ integration of the high resolution PTU sounding. The $C_{n}^{2}$ profile is evaluated using the calculated refractivity. Its variance is estimated in the frequency band $1-3 \mathrm{~Hz}$ (i.e. $1.6-5.0 \mathrm{~m}$ ) within a sliding window of $6 \mathrm{~s}$ corresponding to the radar resolution. The variance is then converted into $C_{n}^{2}$ by assuming a Kolmogorov (isotropic inertial) spectrum.

Weak stratification is observed in the region between 13.9 and $14.7 \mathrm{~km}$ where intermittent turbulent layers are observed by the radar (Fig. 2). The high resolution temperature profile (red curve on Fig. 5) clearly reveals that the fluctuations $\left(C_{n}^{2}\right.$ peaks) can be observed only in regions with increased local stability. Even if turbulence may exist outside such thin (20$50 \mathrm{~m}$ ) regions, the resulting temperature fluctuations are too weak to be measured. Such local stability increase cannot be detected with standard Väisälä soundings (blue curve on Fig. 5). Furthermore, since the altitude of the turbulent layers observed by radar increases with time, one can guess that the altitude of these thin stable layers should also increase. The associated enhancement of $\epsilon_{p}$ appears spurious in such conditions, $\epsilon_{p}$ estimates being inversely proportional to $\omega_{B}^{2}$ (Eq. 19). We therefore discard the data for which the stratification is weak, i.e. $\omega_{B}^{2}<2 \cdot 10^{-4} \mathrm{~s}^{-2}$, that is $28 \%$ of the data obtained on 27 April.

The distributions of dissipation rates, $\epsilon_{k}$ and $\epsilon_{p}$, their ratio, $\epsilon_{p} / \epsilon_{k}$, as well as the inferred $R_{f}$ are displayed in Fig. 6 for the whole data set (27-28/04/98).

The mean value and standard deviation of these estimates are indicated on the plots. Both distributions of $\epsilon_{k}$ and $\epsilon_{p}$ are not symmetrical, possibly due to a threshold detectability effect. Most probable values for $\epsilon_{k}\left(\epsilon_{p}\right)$ are in the range 1$2 \cdot 10^{-4}\left(1-2.5 \cdot 10^{-5}\right) \mathrm{m}^{2} \mathrm{~s}^{-3}$ respectively. Mixing efficiency $\epsilon_{p} / \epsilon_{k}$ and inferred flux Richardson number $R_{f}$, show most probable values in the range $0.05-0.4$ and $0.1-0.3$, respectively. Note that the upper bound of the estimated $R_{f}$ is close to the usual value odtained in most radar studies $(0.25)$, our results therefore suggesting that this value is probably over estimated in most cases. 


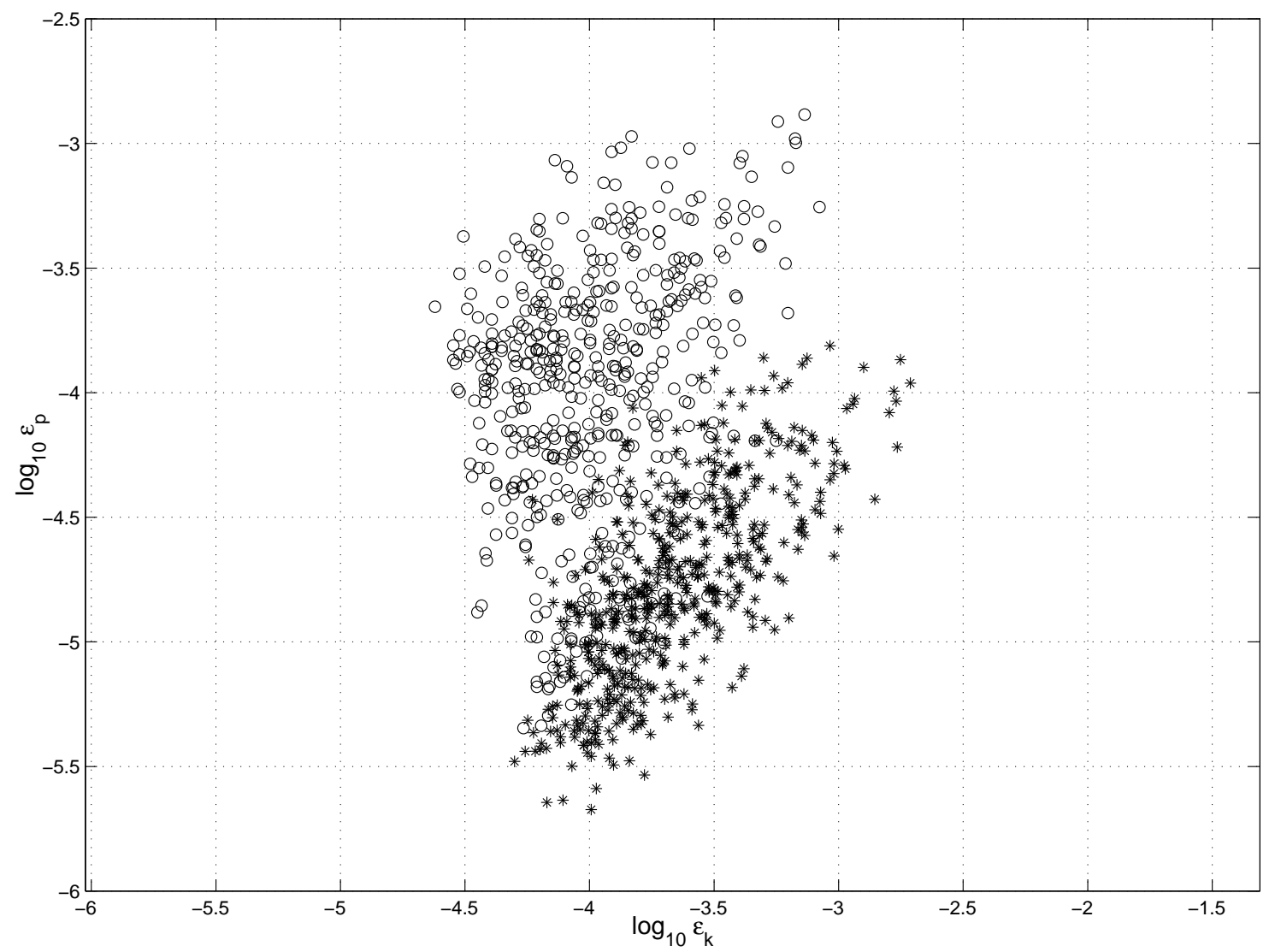

Fig. 4. Scatter plot of $\epsilon_{k}$ versus $\epsilon_{p}$ in case of weak stability (circles) and high stability (stars).

We have also estimated the vertical heat diffusivity (Eq. 7), averaged over the turbulent patches only and time averaged for a given altitude range, from the selected data set $\left(\omega_{B}^{2}>\right.$ $\left.2 \cdot 10^{-4} \mathrm{~s}^{-2}\right)$. The time averaged diffusivity is an estimate of the effective flux, taking into account the intermittency of turbulent patches, divided by a representative gradient (average of two Väisälä soundings). The difference between both estimations is larger than one order of magnitude: the mean value for local heat diffusivity being 0.11 for both data sets, and $6-8 \cdot 10^{-3} \mathrm{~m}^{2} \mathrm{~s}^{-1}$ for the time averaged diffusivity. The local estimate is in good agreement with previously published radar estimations (Fukao et al., 1994; Nastrom and Eaton, 1997). These studies relate the turbulent diffusivity to $\epsilon_{k}$ inferred from the width of the Doppler spectrum. As the first and second moments of the Doppler spectrum do not depend on the space-time intermittency, the inferred diffusivity characterizes the diffusivity within the turbulent layers. On the other hand, the lower estimate agrees rather well with an indirect evaluation of diffusivity within the lower stratosphere obtained by combining aircraft data with a dispersion model (Balluch and Haynes, 1997). This last estimate is also in good agreement with the vertical diffusivity inferred from $\epsilon_{p}$ (Dole and Wilson, 2000) deduced from averaged $C_{n}^{2}$ obtained from medium resolution VHF radar measurements
(375 $\mathrm{m}$ range resolution and 1 hour integration time). In this case, the heat diffusivity is inferred from the radar reflectivity $\eta$ which is an averaged quantity over the observation volume, taking into account the space-time intermittency of the turbulent patches. As a conclusion, the diffusivity inferred from the two methods have different meanings, the local estimation probably overestimating the effective diffusivity (heat flux) due to the space-time intermittency of the turbulent patches.

\section{Conclusions}

We show very high resolution radar and balloon measurements coupled with Väisälä/GPS soundings. We estimate almost independently the dissipation rates of kinetic energy, $\epsilon_{k}$, and potential energy, $\epsilon_{p}$. After discarding the lowest stability regions where the estimate of $\epsilon_{p}$ is likely spurious, we show distributions of dissipation rates of available potential $\left(\epsilon_{p}\right)$ and kinetic $\left(\epsilon_{k}\right)$ energies, and of the inferred flux Richardson number $R_{f}$. Most probable values for dissipation rates of kinetic and potential energy are of the order of $2 \cdot 10^{-4}$ and $4 \cdot 10^{-5}$ respectively. The inferred flux Richardson number, within turbulent patches, seems to be bounded to a limit 


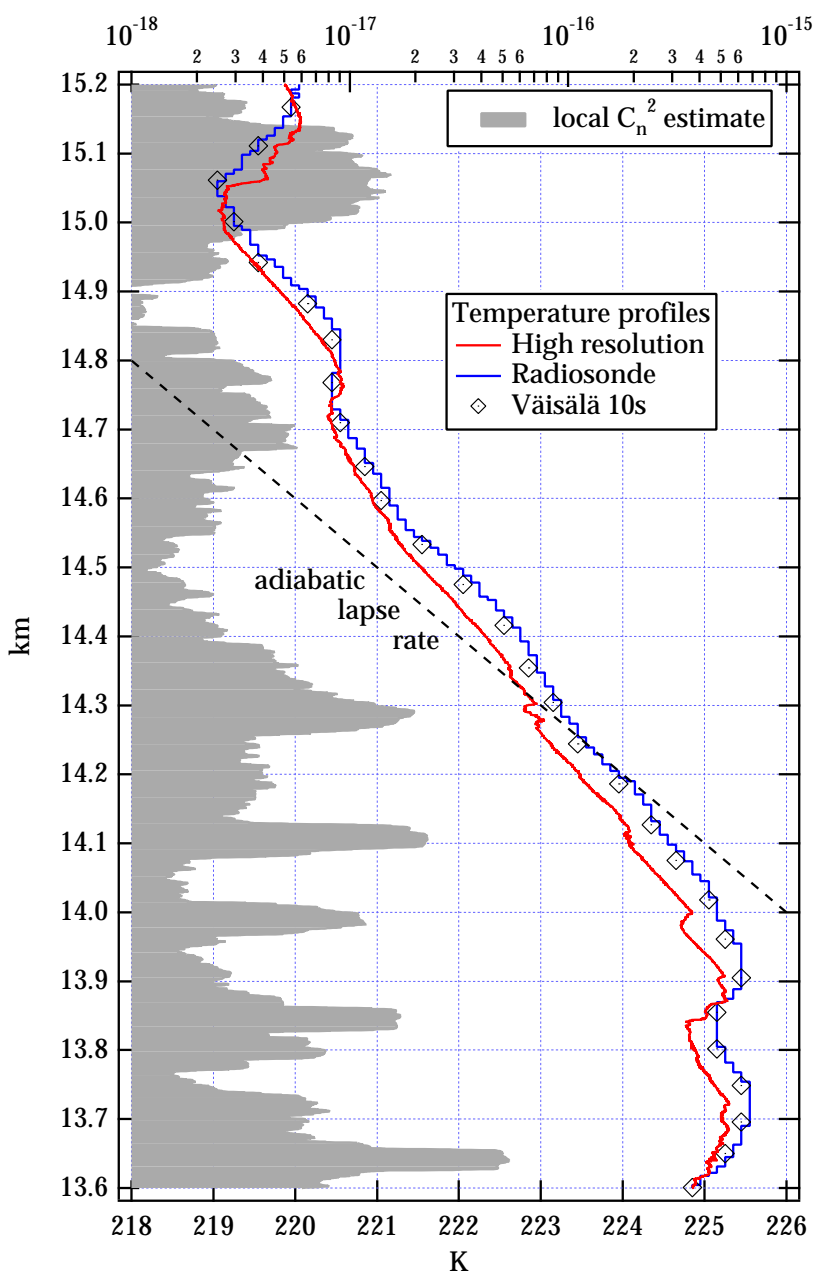

Fig. 5. Simultaneous temperature profiles obtained by the SFT (red) and Väisälä (blue) sensors. The shaded profile shows the $C_{n}^{2}$ estimated from the SFT data.

close to 0.3. We estimate the heat diffusivity from the potential energy dissipation rate, averaged within the turbulent patches and time averaged for a given altitude range. The time averaged vertical diffusivity, less than $10^{-2} \mathrm{~m}^{2} \mathrm{~s}^{-1}$, is observed to be more than one order of magnitude lower than the local diffusivity (within the turbulent patches). The local estimation of diffusivity probably overestimates the effective diffusivity (heat flux) due to the space-time intermittency of the turbulent patches.

Acknowledgement. We thank Richard Ney, Fred Gabizon and Monique Massebeuf for their precious assistance in the providing radar data. The PROUST radar is operated by the Centre d'Étude Terrestre et Planétaire (CETP). We also wish to thank F. Bertin as well as an anonymous reviewer for helpful suggestions and comments.

Topical Editor J.-P. Duvel thanks F.D. Eaton and another referee for their help in evaluating this paper.
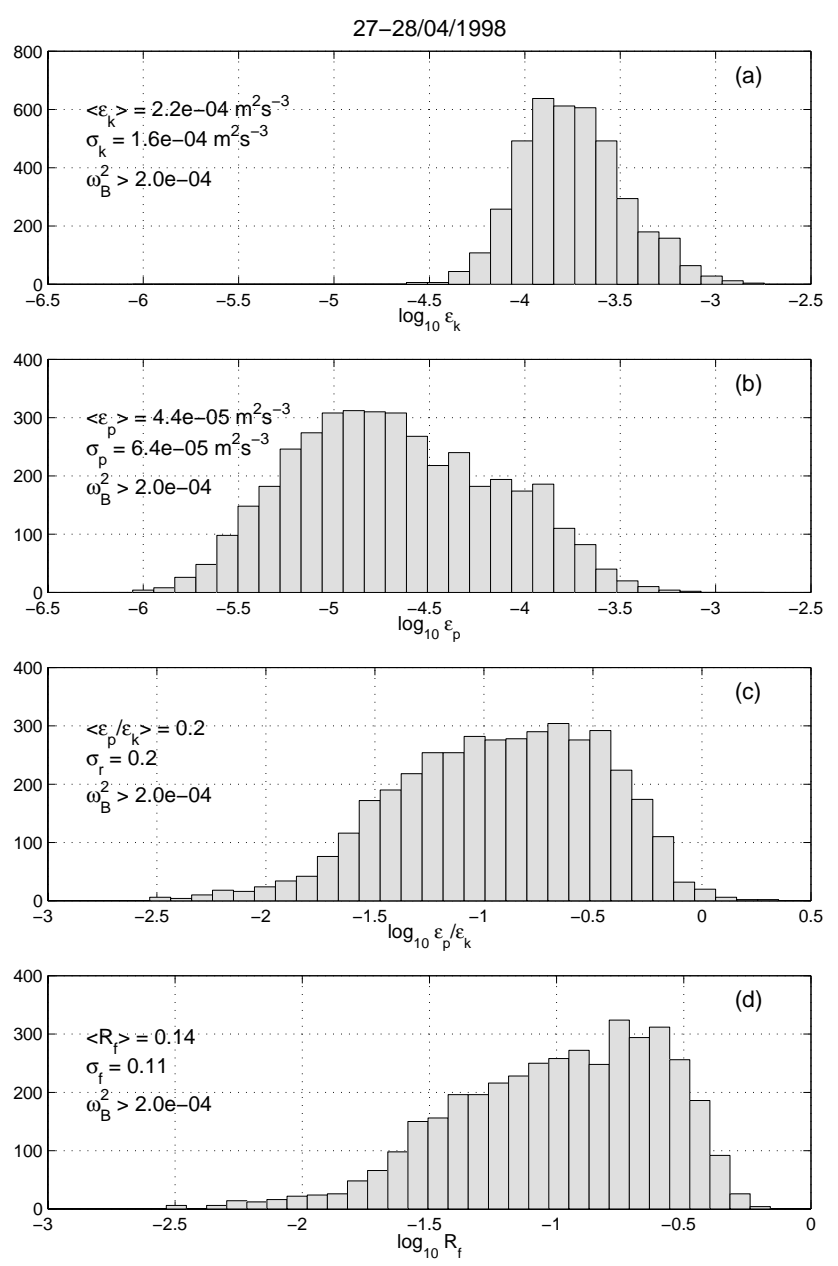

Fig. 6. (a) Dissipation rate of kinetic energy, $\epsilon_{k}$, (b) Dissipation rate of available potential energy, $\epsilon_{p}$, (c) Ratio $\epsilon_{p} / \epsilon_{k}$, (d) Flux Richardson number.

\section{References}

Balluch, M. G. and Haynes, P. H., Quantification of lower stratospheric mixing processes using aircraft data, J. Geophys. Res., 102, 487-504, 1997.

Bertin, F., Crémieu, A., Glass, M., Massebeuf, M., Ney, R., and Petitdidier, M., The Proust radar: first results, Radio Sci., 22, 51-60, 1986.

Dalaudier F., Sidi, C., Crochet, M., and Vernin, J., Direct evidence of "sheets" in the atmospheric temperature field, J. Atmos. Sci., 51, 237-248, 1994.

Delage, D., Bertin, F., Crémieu, A., Massebeuf, M., Ney, R., and Desautez, A., Real time data processing algorithms and first results obtained by the PROUST radar in its final configuration, STEP proceedings of the seventh workshop on Technical and Scientific aspects of MST radars, edited by B. Edwards, SCOSTEP Secr., Boulder, Colo., pp. 209-212, 1996.

Delage, D., Roca, R., Bertin, F., Crémieu, A., Massebeuf, M., and Ney, R., A consistency check of three radar methods for monitoring eddy diffusion and energy dissipation rates through the tropopause, Radio Sci., 32, 757-767, 1997.

Dole, J. and Wilson, R., Estimates of turbulent parameters in the 
lower stratosphere - upper troposphere by radar observations: A novel twist, Geophys. Res. Lett., 27, 2625-2628, 2000.

Doviak R. J. and Zrnić, D. S., Doppler radar and weather observations, Academic Press, San Diego, 1984.

Frehlich, R., Laser scintillation measurements of the temperature spectrum in the atmospheric surface layer, J. Atmos. Sci., 49, 1494-1509, 1992.

Fukao, S., Yamanaka, M., Ao, N., Hocking, W., Sato, T., Yamamoto, M., Nakamura, T., Tsuda, T., and Kato, S., Seasonal variability of vertical diffusivity in the middle atmosphere 1 . Three-year observations by the middle and upper atmosphere radar, J. Geophys. Res., 99, 18973-18987, 1994.

Gage, K., Green, J., and VanZandt, T., Use of Doppler radar for the measurement of atmospheric turbulence parameters from the intensity of clear air radar echo, Radio Sci., 15, 407-416, 1980.

Gargett, A. E. and Moum, J. N., Mixing Efficiencies in turbulent tidal fronts: Results from direct and indirect measurements of density fluxes, J. Phys. Mech. Oceanogr., 25, 2583-2608, 1995.

Hill, R. J., Models of the scalar spectrum for turbulent advection, J. Fluid Mech., 88, 541-562, 1978a.

Hill, R. J., Spectra of fluctuations in refractivity, temperature, humidity, and the temperature-humidity cospectrum in the inertial and dissipation range, Radio Sci., 13, 953-961, 1978 b.

Hocking, W. K., On the extraction of atmospheric turbulence pa- rameter from radar backscatter Doppler spectra-I. Theory, J. Atmos. Terr. Phys., 45, 89-102, 1983.

Hocking, W. K., Measurement of turbulent energy dissipation rates in the middle atmosphere by radar techniques: A review, Radio Sci., 20, 1403-1422, 1985.

Monin A. S. and Yaglom, A. M., Statistical Fluid Mechanics: Mechanics of turbulence, MIT press, 1975.

Nastrom, G. D. and Eaton, F. D., Turbulence eddy dissipation rates from radar observations at 5-20 km at White Sands Missile Range, New Mexico, J. Geophys. Res., 102, 19495-19505, 1997.

Ottersten, H., Radar backscattering from the turbulent clear atmosphere, Radio Sci., 4, 1251-1255, 1969.

Petitdidier, M., Desautez, A., Glass, M., and Penazzi, G., A decoder for a $30 \mathrm{~m}$ height resolution ST radar, Radio Sci., 20, 1141-1145, 1985.

Sato T. and Woodman, R. F., Fine altitude resolution of stratospheric turbulent layers by the Arecibo $430 \mathrm{MHz}$ radar, J. Atmos. Sci., 39, 2546-2552, 1982.

Tatarski, V. I., Wave propagation in a turbulent medium, McGrawHill, New York, 1961.

Weinstock, J., On the theory of turbulence in the buoyancy subrange of stably stratified flows, J. Atmos. Sci., 35, 634-649, 1978.

Weinstock, J., Using radar to estimate dissipation rates in thin layers of turbulence, Radio Sci., 16, 1401-1406, 1981. 